\title{
A Modular, Enantioselective Synthesis of Resolvins D3, E1, and Hybrids
}

\author{
Felix Urbitsch, Bryony L. Elbert, Josep Llaveria, Penelope E. Streatfeild, and Edward A. Anderson*
}

Cite This: Org. Lett. 2020, 22, 1510-1515

Read Online

ABSTRACT: Resolvins D3 and E1 are important signaling molecules in the resolution of inflammation. Here, we report a convergent and flexible strategy to prepare these natural products using Hiyama-Denmark coupling of five- and six-membered cyclic alkenylsiloxanes to connect three resolvin fragments, and control the stereochemistry of the natural product $(Z)$-alkenes. The modular nature of this approach enables the synthesis of novel resolvin hybrids, opening up opportunities for more-extensive investigations of resolvin biology.

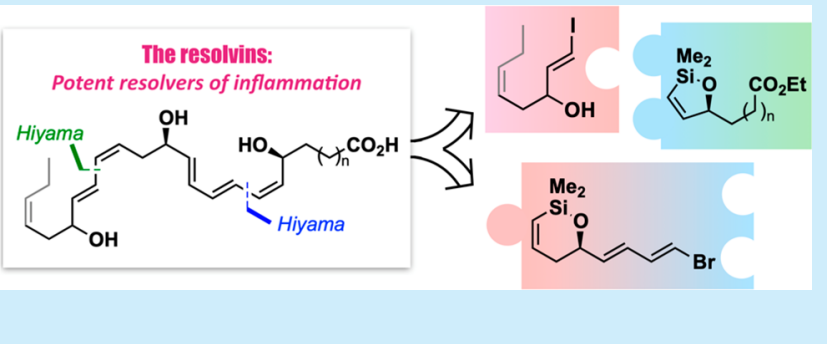

$\mathrm{T}$ he resolution of inflammation is a complex process regulated by a host of different signaling molecules, including the resolvin, protectin, and maresin pro-resolving mediators (e.g., 1-4, Figure 1). ${ }^{1}$ These polyunsaturated fatty
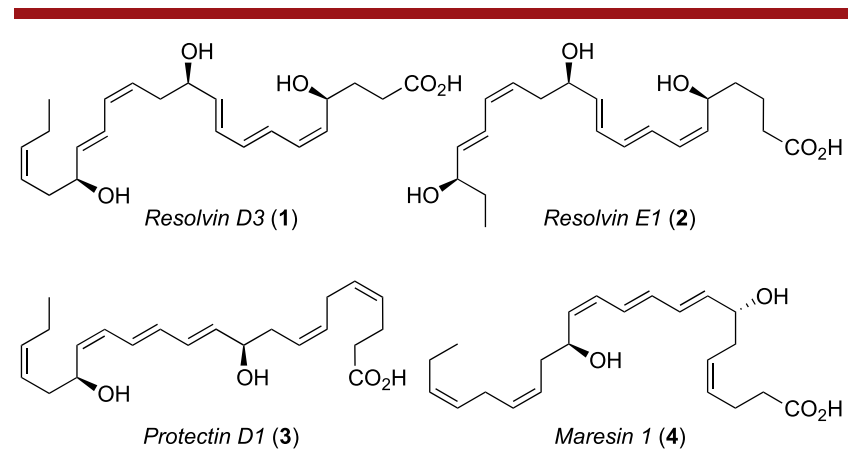

Figure 1. Pro-resolving natural products: Resolvin D3 (RvD3) (1), resolvin E1 (RvE1) (2), protectin D1 (3), and maresin 1 (4).

acids, which display nanomolar to picomolar bioactivity, stimulate a cascade of cellular resolution events involving the reduction of polymorphonuclear neutrophil infiltration and the initiation of macrophage clearance of apoptotic cells. Since numerous diseases are associated with chronic or excessive inflammation (such as cardiovascular diseases, asthma, diabetes, and neurodegenerative diseases), ${ }^{2}$ there is great interest in the synthesis of these natural products in order to develop a deeper understanding of their individual roles., ${ }^{\text {b3 } 3}$ Resolvins D3 (RvD3, 1) and E1 (RvE1, 2) are typical examples of these polyhydroxylated lipid mediators, with the former being the most potent member of the family. ${ }^{4}$

Subsequent to their initial discovery and isolation by Serhan et al., ${ }^{5}$ the structures and stereochemistry of RvD3 and RvE1 were confirmed by Petasis and Serhan via total synthesis. ${ }^{6}$ Both contain a $(Z, E)$-diene and a $(Z, E, E)$-triene motif; in these previous approaches, the isomerization-prone $(Z)$-alkene of the triene unit was revealed in the final step via semireduction of the corresponding enynes with $\mathrm{Zn} / \mathrm{Ag} / \mathrm{Cu}$. This tactic has been adopted by others for related resolvins, ${ }^{7}$ and has enabled the in vivo testing of the natural products. A further synthesis of resolvin E1 was disclosed in which the $(Z)$-configured double bonds were introduced from a $(Z)$-alkenyl bromide via Suzuki coupling, and from a Wittig reaction. ${ }^{8}$

Here, we describe an alternative strategy in which late-stage Hiyama-Denmark cross-coupling ${ }^{9}$ of cyclic alkenylsiloxanes is used to specifically control the stereochemistry of the $(Z)$ alkenes in the resolvin polyene motifs. ${ }^{10}$ Disconnection at these alkenes (Scheme 1a) reveals five- and six-membered cyclic alkenylsiloxanes (5/6 and 7/8, respectively), along with two alkenyl iodide resolvin "tails" (9 and 10). RvD3 and RvE1 are ideal candidates for this approach, as in previous work (Scheme 1b), ${ }^{10 a}$ we established that five-membered cyclic diethyl alkenylsiloxanes, prepared by Lindlar hydrogenation of the corresponding alkynylsiloxanes, undergo Hiyama coupling more rapidly and under distinct conditions (i.e., KOTMS as activator), compared to six-membered siloxanes (which require fluoride as the coupling promoter). We subsequently found that the more reactive cyclic dimethyl alkenylsiloxanes can be accessed using benzyldimethylsilanes as latent silanols (Scheme 1c), with the cyclic siloxane revealed through fluoride- or (for five-membered rings) base-mediated debenzylation. $^{11}$

Received: January 8, 2020

Published: February 7, 2020 
Scheme 1. (a) A Modular Strategy toward Resolvins D3 and E1; (b) Selectivity in the Cross-Coupling of Cyclic Diethylalkenylsiloxanes; (c) Synthesis of Cyclic Dimethylalkenylsiloxanes from Benzyldimethylsilanes

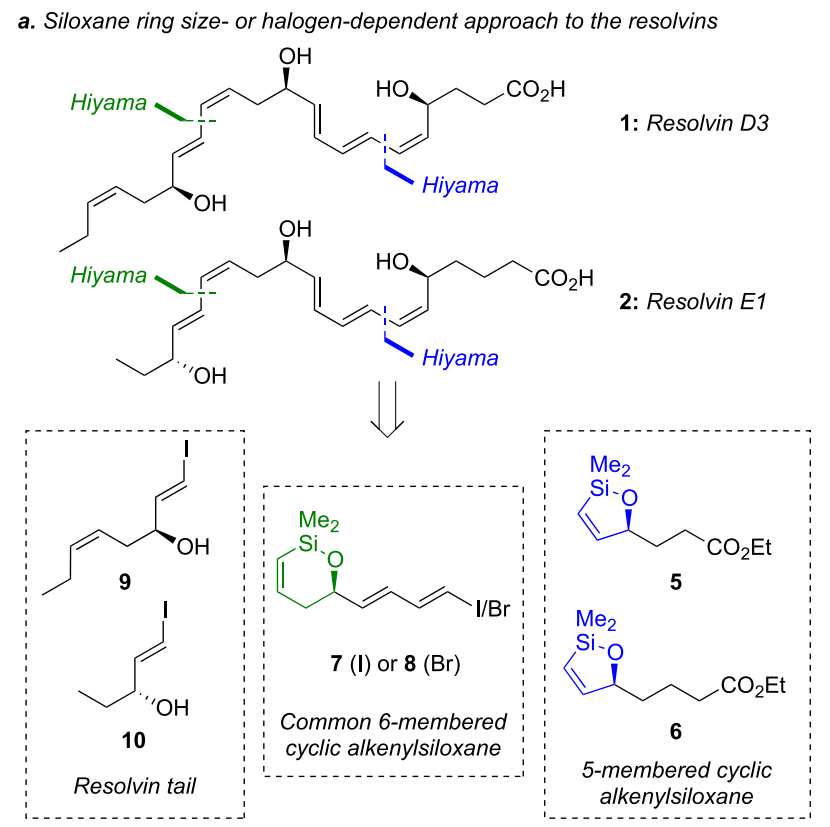

b. Previous work: Ring size selective cross-coupling ${ }^{11 a}$

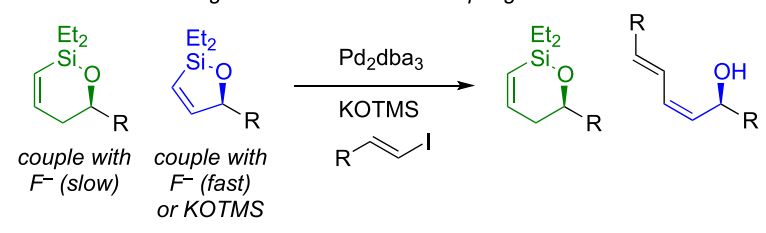

c. Previous work: Cyclic dimethylsiloxanes from benzyldimethylsilanes ${ }^{12}$

$$
\mathrm{BnMe}_{2} \mathrm{Si}
$$

Based on this ring size-dependent reactivity, we planned a "head-to-tail" coupling strategy involving initial selective coupling of five-membered siloxanes $5 / 6$ with dienyl halides 7 or 8 , followed by coupling of the residual six-membered siloxane with iodides 9 or $\mathbf{1 0}$. Alternatively, we envisaged a "tail-to-head" approach, in which control would be achieved through differentiated rates of oxidative addition (I vs Br) in the initial coupling of iodides 9 or $\mathbf{1 0}$ with the central bromodiene siloxane 8 . The modular nature of the synthesis would allow access to both the natural products and to novel resolvin analogues by mixing components from the different synthesis streams. As very few unnatural resolvins have been studied, this route could open up opportunities for a wider exploration of the effects of chain length, stereochemistry, and the nature of the head/tail functionality on resolvin biology. ${ }^{12}$

The synthesis of the $\mathrm{C} 1-\mathrm{C} 6$ and $\mathrm{C} 1-\mathrm{C} 7$ "head" fragments 5 and $\mathbf{6}$ (Scheme 2, required for RvD3 and RvE1, respectively) began with the addition of benzyl(ethynyl)dimethylsilane to the commercially available acid chlorides 11 and 12 . The yields of these reactions proved quite dependent on chain length, with 13 formed in 64\% yield, but homologue 14 in just $26 \%$ yield; fortunately, the latter could be improved to $52 \%$ by use of an alkynylzinc. ${ }^{13}$ The resulting ketones were converted to
Scheme 2. Synthesis of Resolvin Building Blocks 5-10
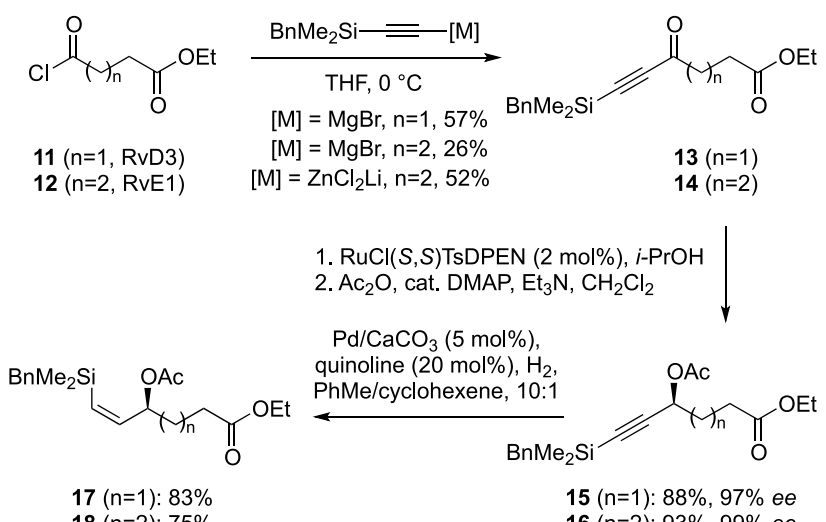
$18(n=2): 75 \% \quad 16(n=2): 93 \%, 99 \%$ ee

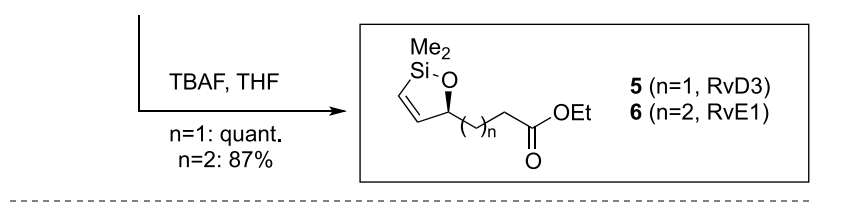

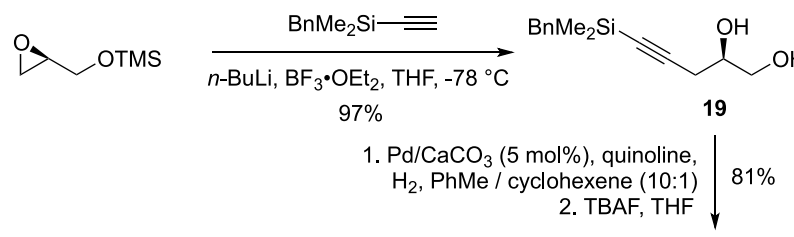
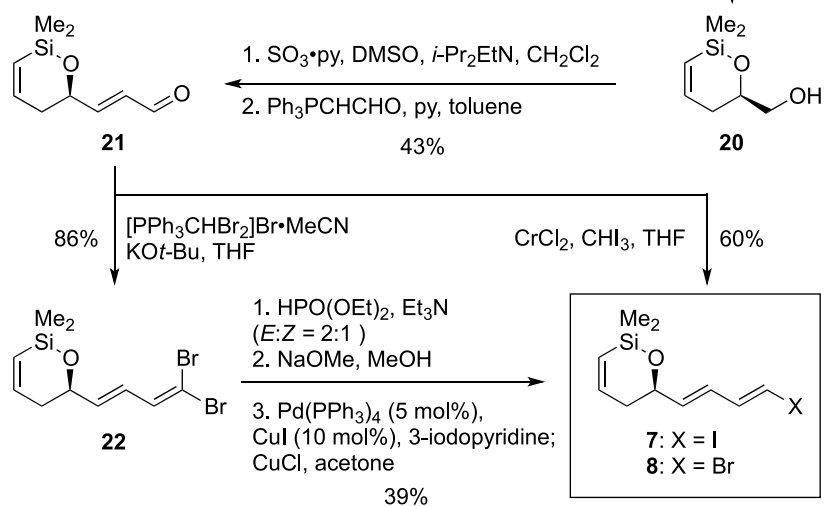

$39 \%$
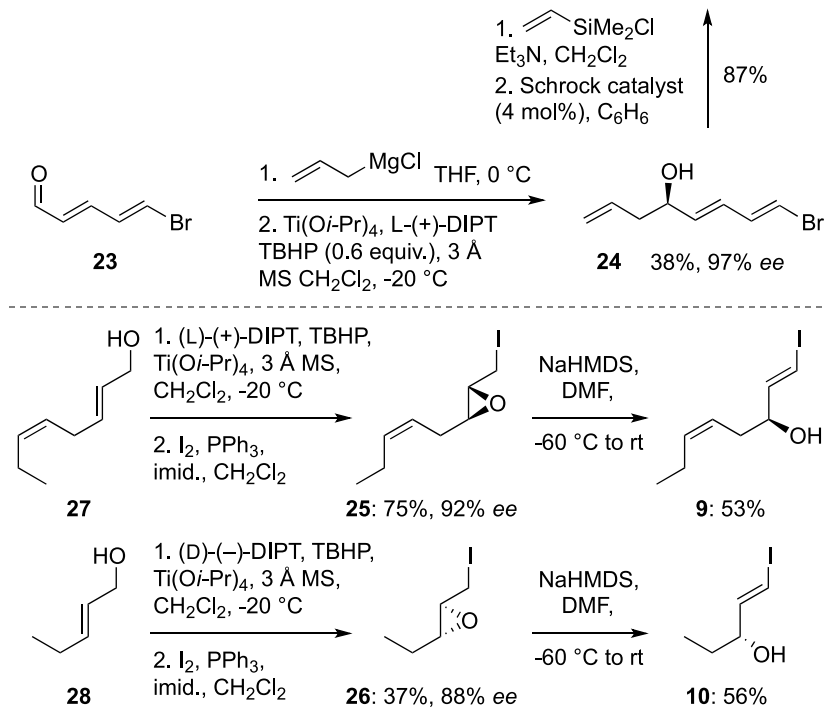

the enantioenriched propargylic acetates 15 and 16 through Noyori asymmetric transfer hydrogenation (97\%-99\% enan- 
tiomeric excess (ee)), ${ }^{14}$ followed by esterification. ${ }^{15}$ Semihydrogenation to the $(Z)$-benzyldimethyl alkenylsilanes 17 and 18 proceeded with high yield and selectivity $(75 \%-83 \%, Z: E>$ 20:1). These products were treated with TBAF, which effected debenzylation, in situ deacetylation, and cyclization to give the cyclic five-membered siloxanes 5 and $\mathbf{6}$ in excellent yields.

Synthesis of the "middle" fragments 7 and 8 (Scheme 2, common to both RvD3 and RvE1) initially utilized a chiral pool strategy. The addition of lithium benzyldimethylsilylacetylide to TMS-protected (S)-glycidol afforded diol 19 in $98 \%$ yield, which was carried through the Lindlar hydrogenation/ cyclization sequence $^{10 a}$ to give the six-membered cyclic alkenylsiloxane 20 (81\%). Parikh-Doering oxidation afforded an unstable aldehyde, which was used directly in a Wittig olefination to give enal 21, which displayed greater stability and could be purified by chromatography. Here, a serendipitous discovery was made: residual pyridine from the ParikhDoering oxidation improved the yield of the olefination from $32 \%$ to $52 \%$ ( $43 \%$ overall). 21 was converted to dienyl iodide 7 via Takai iodoolefination $(60 \%, E: Z=5: 1)$. Synthesis of the equivalent bromide $\mathbf{8}$ was achieved in two steps, consisting of Ramirez olefination (22, 86\%), followed by Hirao monodebromination using dimethyl phosphite. ${ }^{16}$ This latter reaction is known to exhibit variable selectivity for conjugated systems, ${ }^{17}$ and indeed the product bromodiene was obtained as a $2: 1 \quad(E, E):(E, Z)$ mixture. Variation of solvent or temperature did not affect this ratio, and while the bulkier diisopropyl phosphite offered a modest improvement $(E: Z=$ 2.5:1), the conversion decreased significantly. Fortunately, the undesired $(E, Z)$-dienyl bromide could be removed by elimination of $\mathrm{HBr}$ (refluxing $\mathrm{NaOMe}){ }^{18}$ followed by Sonogashira coupling of the resulting enyne with 2iodopyridine. Despite the obstacles encountered, this sequence does illustrate the capacity of the six-membered cyclic dimethylsiloxane to survive a range of reaction conditions.

To overcome the limitations of this route, a shorter synthesis was developed, exploiting the Denmark ring-closing metathesis approach to cyclic dimethylsiloxanes. ${ }^{10 \mathrm{~b}-\mathrm{f}}(E, E)$-5-bromopentadienal 23 was readily accessed from $\mathrm{SO}_{3} \cdot$ py by alkaline hydrolysis and bromination. ${ }^{19}$ The addition of allylmagnesium bromide, followed by Sharpless resolution ( $98 \%$ ee), ${ }^{20}$ afforded enantio-enriched alcohol 24. Formation of an intermediate vinyldimethylsilyl ether set the stage for ring-closing metathesis mediated by the Schrock catalyst, ${ }^{10 \mathrm{~b}-\mathrm{f}}$ which proceeded in excellent yield ( $87 \%$ over two steps).

The resolvin "tail" fragments, destined for coupling with the central six-membered cyclic siloxane, were prepared via base mediated ring-opening of $\alpha$-iodoepoxides 25 and 26 as developed by Spur $^{21}$ and Nakata (Scheme 2), ${ }^{22}$ which selectively afforded the desired (E)-iodoalkenes 9 and $\mathbf{1 0}$ respectively. The configuration of these epoxides was set by Sharpless epoxidation of the corresponding allylic alcohols 27 and 28 . $^{23}$

With all key fragments in hand, attention turned to assembly of the resolvin framework. We first studied the "head-to-tail" strategy which would rely on an enhanced rate of transmetalation for the five-membered siloxane over the sixmembered siloxane in the initial cross-coupling, as had been observed in our earlier work with equivalent diethylsiloxanes (see Scheme 1, eq 1). ${ }^{10 a}$ Indeed, we were pleased to find that model fluoride-promoted couplings of iodoalkene 29 with substrates 30 and 31 (Scheme 3, eq 1) revealed significantly more rapid and higher yielding coupling of the five-membered
Scheme 3. Evaluation of Cross-Coupling Strategies

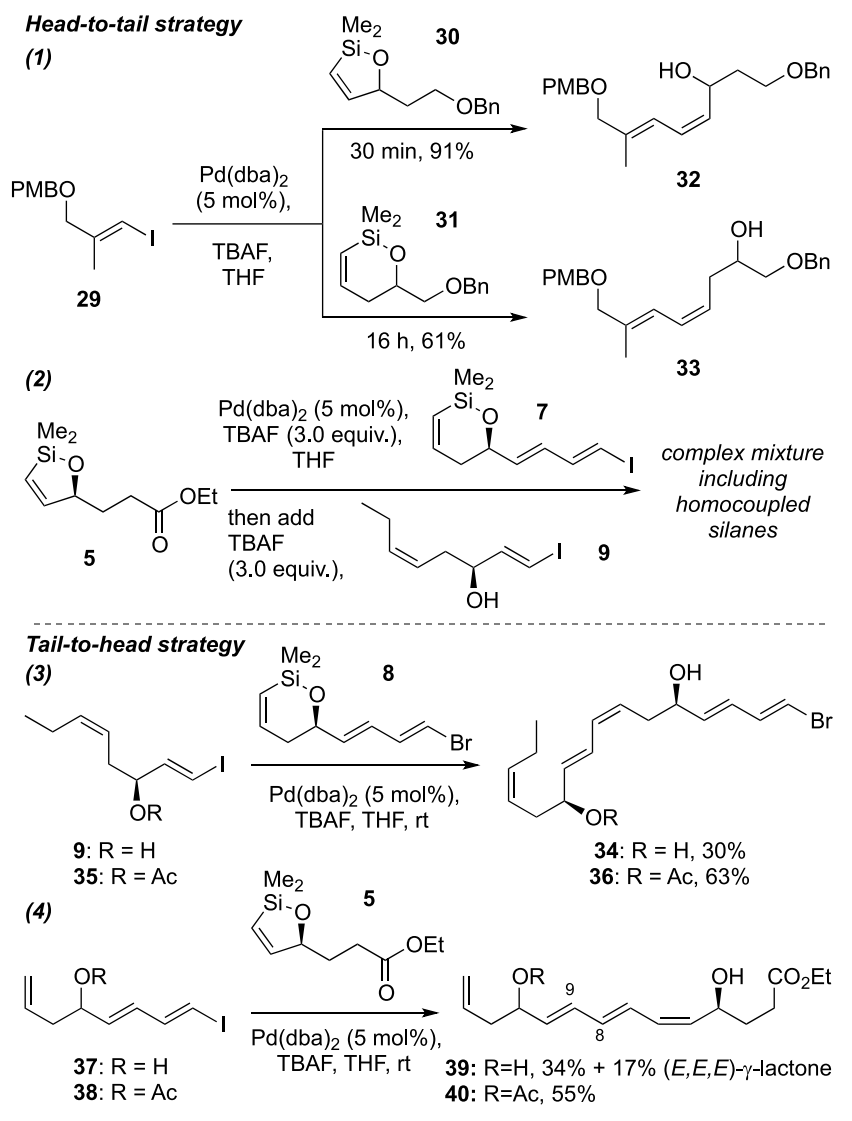

ring siloxane 30 (91\%). However, attempts to translate this reactivity difference to selective coupling of $\mathbf{5}$ in the presence of the six-membered siloxane in 7 met with failure (Scheme 3, eq 2), whether using fluoride or KOTMS as the promoter, and with simultaneous or sequential addition of the reactants; side reactions including desilylation, homocoupling, and ester hydrolysis were observed in a variety of model studies. It appeared that while less reactive toward transmetalation, the six-membered ring was nonetheless susceptible to rapid ring opening under the reaction conditions, compromising the ability of the coupling promoter to mediate selective coupling of the five-membered ring.

Examination of the "tail-to-head" strategy proved more fruitful. This approach relies on selectivity in the oxidative addition of the resolvin vinyl iodide "tails" $(9 / 10)$ over the dienyl bromide 8 in the central fragment. We first studied the coupling of RvD3 iodide 9 with 8 (Scheme 3, eq 3); this coupling proved sluggish and low yielding (30\%), and resulted in the formation of a byproduct tentatively assigned as homocoupling of iodide $9 .^{24}$ However, reaction of the acetate derivative of the allylic alcohol (35) proceeded at a significantly higher rate, and delivered product 36 in higher yield $(63 \%)$ and without iodide dimerization. The benefit of acetylation was reinforced in an equivalent model coupling of dienyl iodide 37 with RvE1 "head" siloxane 5 (Scheme 3, eq 4). Reaction of the free alcohol 37 (to give 39) led to competing formation of a byproduct assigned as an isomerized $\gamma$-lactone; ${ }^{24}$ use of the acetate $\mathbf{3 8}$ derivative suppressed this side reaction and proceeded in higher yield (38 $\rightarrow 40,55 \%)$. We suggest that the free allylic alcohols in 9 or 37 may 
Scheme 4. Synthesis of Resolvins D3, E1, and Hybrids ${ }^{a}$

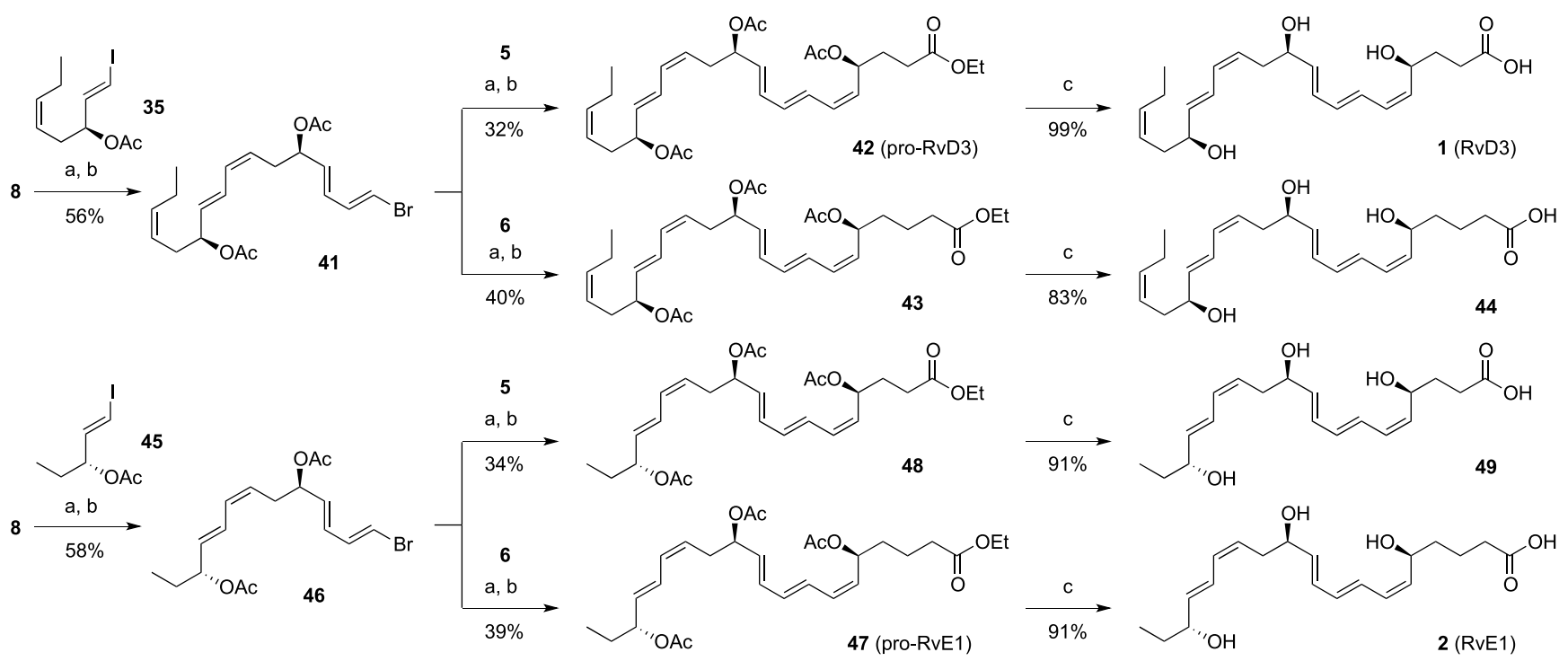

${ }^{a}$ Reagents and conditions: (a) Pd(dba) $)_{2}\left(5 \mathrm{~mol} \%\right.$ ), TBAF (3 equiv), THF, 16 h; (b) $\mathrm{Ac}_{2} \mathrm{O}$, py, $\mathrm{CH}_{2} \mathrm{Cl}_{2} ;$ (c) $\mathrm{LiOH}, \mathrm{THF} / \mathrm{H}_{2} \mathrm{O}(1: 1)$.

interfere with the efficiency of coupling by moderating the reactivity of the fluoride activator.

Irrespective of the basis of this beneficial effect, the Hiyama-Denmark coupling had now been validated for the formation of both $\mathrm{C}-\mathrm{C}$ bonds, and our attention turned to completion not only of the natural resolvins 1 and 2, but also resolvin hybrids by mixing different natural product building blocks. As such, coupling of $\mathbf{8}$ and $\mathbf{3 5}$ (Scheme 4), followed by acetylation, afforded dienyl bromide 41 (53\%). This was coupled with siloxanes $\mathbf{5}$ and $\mathbf{6}$ to give product alcohols that were immediately acetylated (42 and 43, respectively; $32 \%-$ $40 \%)$ - in part to aid purification from a $\gamma$-lactone formed from cyclization of the $\gamma$-hydroxyester headgroup, ${ }^{25}$ but also to impart stability toward long-term storage of these "proresolvins", compared to the natural products. Resolvin D3 $(\mathbf{1})^{26}$ and the RvD3/E1 hybrid 44 were revealed in near quantitative yield upon treatment with lithium hydroxide. Similar coupling of 8 with iodoalkene acetate 45 gave the tetraene 46 after acetylation (58\%). Coupling of 46 with the two headgroup siloxanes and acetylation now afforded triacetate pro-RvE1 47 and hybrid 48. Again, these could be saponified in high yield on treatment with aqueous lithium hydroxide to afford resolvin E1 $(2)^{27}$ and the RvE1/D3 hybrid 49.

In conclusion, resolvins D3 and E1 were prepared in 12 steps in the longest linear sequence ( $\sim 20$ steps total), employing Hiyama cross-coupling of cyclic alkenylsiloxanes in key fragment union transformations. The modular and convergent nature of the route also enabled the synthesis of new resolvin analogues. These enantioselective syntheses open up opportunities for a wider study of the role of these $(Z)$ alkenyl polyene natural products in inflammatory response pathways; studies to this end are ongoing in our group.

\section{ASSOCIATED CONTENT}

\section{Supporting Information}

The Supporting Information is available free of charge at https://pubs.acs.org/doi/10.1021/acs.orglett.0c00089.
Experimental details and copies of ${ }^{1} \mathrm{H}$ and ${ }^{13} \mathrm{C}$ NMR spectra (PDF)

\section{AUTHOR INFORMATION}

Corresponding Author

Edward A. Anderson - Chemistry Research Laboratory, Oxford OX1 3TA, United Kingdom; O orcid.org/0000-0002-4149-

0494; Email: edward.anderson@chem.ox.ac.uk

\section{Authors}

Felix Urbitsch - Chemistry Research Laboratory, Oxford OX1 3TA, United Kingdom

Bryony L. Elbert - Chemistry Research Laboratory, Oxford OX1 3TA, United Kingdom

Josep Llaveria - UCB Pharma, Ltd., Slough SL1 3WE, United Kingdom; ○ orcid.org/0000-0003-2260-3657

Penelope E. Streatfeild - Chemistry Research Laboratory, Oxford OX1 3TA, United Kingdom

Complete contact information is available at: https://pubs.acs.org/10.1021/acs.orglett.0c00089

Notes

The authors declare no competing financial interest.

\section{ACKNOWLEDGMENTS}

F.U. thanks the EPSRC Centre for Doctoral Training in Synthesis for Biology and Medicine (No. EP/L015838/1) for a studentship, generously supported by AstraZeneca, Diamond Light Source, Defence Science and Technology Laboratory, Evotec, GlaxoSmithKline, Janssen, Novartis, Pfizer, Syngenta, Takeda, UCB and Vertex. E.A.A. thanks the EPSRC for additional support (No. EP/M019195/1). We thank the UCB Global Analytical Science team for assistance with HPLC purification of the resolvin triacetates.

\section{REFERENCES}

(1) (a) Serhan, C. N. Pro-resolving lipid mediators are leads for resolution physiology. Nature 2014, 510, 92-101. (b) Serhan, C. N.; 
Petasis, N. A. Resolvins and Protectins in Inflammation Resolution. Chem. Rev. 2011, 111, 5922-5943. (c) Fullerton, J. N.; Gilroy, D. W. Resolution of inflammation: a new therapeutic frontier. Nat. Rev. Drug Discovery 2016, 15, 551-567. (d) Buckley, C. D.; Gilroy, D. W.; Serhan, C. N. Proresolving Lipid Mediators and Mechanisms in the Resolution of Acute Inflammation. Immunity 2014, 40, 315-327. (e) Tang, S.; Wan, M.; Huang, W.; Stanton, R. C.; Xu, Y. Maresins: Specialized Proresolving Lipid Mediators and Their Potential Role in Inflammatory-Related Diseases. Mediators Inflammation 2018, 2018, 2380319.

(2) (a) Hansson, G. K.; Libby, P. The immune response in atherosclerosis: a double-edged sword. Nat. Rev. Immunol. 2006, 6, 508-519. (b) Kumar, V.; Abbas, A. K.; Fausto, N.; Robbins, S. L.; Cotran, R. S. Robbins and Cotran Pathologic Basis of Disease; Elsevier Saunders, 2005.

(3) Vik, A.; Dalli, J.; Hansen, T. V. Recent advances in the chemistry and biology of anti-inflammatory and specialized pro-resolving mediators biosynthesized from n-3 docosapentaenoic acid. Bioorg. Med. Chem. Lett. 2017, 27, 2259-2266.

(4) Dalli, J.; Winkler, J. W.; Colas, R. A.; Arnardottir, H.; Cheng, C.Y. C.; Chiang, N.; Petasis, N. A.; Serhan, C. N. Resolvin D3 and Aspirin-Triggered Resolvin D3 Are Potent Immunoresolvents. Chem. Biol. 2013, 20, 188-201.

(5) Serhan, C. N.; Clish, C. B.; Brannon, J.; Colgan, S. P.; Chiang, N.; Gronert, K. Novel functional sets of lipid-derived mediators with antiinflammatory actions generated from omega-3 fatty acids via cyclooxygenase 2-nonsteroidal antiinflammatory drugs and transcellular processing. J. Exp. Med. 2000, 192, 1197-1204.

(6) (a) Winkler, J. W.; Uddin, J.; Serhan, C. N.; Petasis, N. A. Stereocontrolled Total Synthesis of the Potent Anti-inflammatory and Pro-resolving Lipid Mediator Resolvin D3 and Its Aspirin-Triggered 17R-Epimer. Org. Lett. 2013, 15, 1424-1427. (b) Arita, M.; Bianchini, F.; Aliberti, J.; Sher, A.; Chiang, N.; Hong, S.; Yang, R.; Petasis, N. A.; Serhan, C. N. Stereochemical assignment, antiinflammatory properties, and receptor for the omega-3 lipid mediator resolvin E1. J. Exp. Med. 2005, 201, 713-722.

(7) (a) Rodriguez, A. R.; Spur, B. W. Total synthesis of Resolvin D1, a potent anti-inflammatory lipid mediator. Tetrahedron Lett. 2012, 53, 6990-6994. (b) Li, J.; Leong, M. M.; Stewart, A.; Rizzacasa, M. A. Total synthesis of the endogenous inflammation resolving lipid resolvin D2 using a common lynchpin. Beilstein J. Org. Chem. 2013, 9, 2762-2766. (c) Morita, M.; Wu, S.; Kobayashi, Y. Stereocontrolled synthesis of resolvin D1. Org. Biomol. Chem. 2019, 17, 2212-2222. (d) Winkler, J. W.; Orr, S. K.; Dalli, J.; Cheng, C.-Y. C.; Sanger, J. M.; Chiang, N.; Petasis, N. A.; Serhan, C. N. Resolvin D4 stereoassignment and its novel actions in host protection and bacterial clearance. Sci. Rep. 2016, 6, 18972. (e) Rodríguez, A. R.; Spur, B. W. First total synthesis of $7(S), 16(R), 17(S)$-Resolvin D2, a potent anti-inflammatory lipid mediator. Tetrahedron Lett. 2004, 45, 8717-8720.

(8) (a) Ogawa, N.; Kobayashi, Y. Total synthesis of resolvin E1. Tetrahedron Lett. 2009, 50, 6079-6082. (b) Sciavolino, F. C.; Matthias, G.; Van Zandt, M. C.; Jadmann, G. E., Jr.; Dworak, J. J. Compositions and methods relating to salts of specialized proresolving mediators of inflammation. International Patent No. WO2017210604A1, 2017. (c) Petasis, N. A. Trihydroxy Polyunsaturated Eicosanoids. U.S. Patent Application No. 2003236423, 2003. (d) Allard, M.; Barnes, K.; Chen, X.; Cheung, Y.-Y.; Duffy, B.; Heap, C.; Inthavongsay, J.; Johnson, M.; Krishnamoorthy, R.; Manley, C.; Steffke, S.; Varughese, D.; Wang, R.; Wang, Y.; Schwartz, C. E. Total synthesis of Resolvin E1. Tetrahedron Lett. 2011, 52, 2623-2626. (e) Amin, R.; Chen, J.-X.; Cotterill, I. C.; Emrich, D.; Ganley, D.; Khmelnitsky, Y. L.; McLaws, M. D.; Michels, P. C.; Schwartz, C. E.; Thomas, D.; Yan, J.; Yang, Q. Improved Synthesis of the C16-C20 Segment of Resolvin E1 Using Enantioselective Ketone Reduction and Lipase-Catalyzed Resolution. Org. Process Res. Dev. 2013, 17, 915-920.

(9) (a) Sore, H. F.; Galloway, W. R. J. D.; Spring, D. R. PalladiumCatalysed Cross-Coupling of Organosilicon Reagents. Chem. Soc. Rev. 2012, 41, 1845-1866. (b) Nakao, Y.; Hiyama, T. Silicon-Based
Cross-Coupling Reaction: An Environmentally Benign Version. Chem. Soc. Rev. 2011, 40, 4893-4901. (c) Denmark, S. E.; Liu, J. H. C. Silicon-Based Cross-Coupling Reactions in the Total Synthesis of Natural Products. Angew. Chem., Int. Ed. 2010, 49, 2978-2986. (d) Denmark, S. E.; Regens, C. S. Palladium-Catalyzed CrossCoupling Reactions of Organosilanols and Their Salts: Practical Alternatives to Boron- and Tin-Based Methods. Acc. Chem. Res. 2008, 41, 1486-1499. (e) Denmark, S. E.; Sweis, R. F. Organosilicon Compounds in Cross-Coupling Reactions. In Metal-Catalyzed CrossCoupling Reactions; de Meijere, A., Diederich, F., Eds.; Wiley-VCH, 2004; pp 163-216.

(10) (a) Elbert, B. L.; Lim, D. S. W.; Gudmundsson, H. G.; O'Hanlon, J. A.; Anderson, E. A. Synthesis of Cyclic Alkenylsiloxanes by Semihydrogenation: A Stereospecific Route to (Z)-Alkenyl Polyenes. Chem. - Eur. J. 2014, 20, 8594-8598. (b) Denmark, S. E.; Muhuhi, J. M. Development of a General, Sequential, Ring-Closing Metathesis/Intramolecular Cross-Coupling Reaction for the Synthesis of Polyunsaturated Macrolactones. J. Am. Chem. Soc. 2010, 132, 11768-11778. (c) Denmark, S. E.; Yang, S. M. Sequential RingClosing Metathesis/Pd-Catalyzed, Si-Assisted Cross-Coupling Reactions: General Synthesis of Highly Substituted Unsaturated Alcohols and Medium-Sized Rings Containing a 1,3-cis-cis Diene Unit. Tetrahedron 2004, 60, 9695-9708. (d) Denmark, S. E.; Yang, S.-M. Intramolecular Silicon-Assisted Cross-Coupling Reactions: General Synthesis of Medium-Sized Rings Containing a 1,3-cis-cis Diene Unit. J. Am. Chem. Soc. 2002, 124, 2102-2103. (e) Denmark, S. E.; Yang, S. M. Sequential Ring-Closing Metathesis and Silicon-Assisted CrossCoupling Reactions: Stereocontrolled Synthesis of Highly Substituted Unsaturated Alcohols. Org. Lett. 2001, 3, 1749-1752. (f) Chang, S.; Grubbs, R. H. A Simple Method to Polyhydroxylated Olefinic Molecules Using Ring-Closing Olefin Metathesis. Tetrahedron Lett. 1997, 38, 4757-4760. (g) Denmark, S. E.; Yang, S. M. Intramolecular Silicon-Assisted Cross-Coupling: Total Synthesis of (+)-Brasilenyne. J. Am. Chem. Soc. 2002, 124, 15196-15197. (h) Denmark, S. E.; Yang, S. M. Total Synthesis of (+)-Brasilenyne. Application of an Intramolecular Silicon-Assisted Cross-Coupling Reaction. J. Am. Chem. Soc. 2004, 126, 12432-12440. (i) Trost, B. M.; Ball, Z. T.; Laemmerhold, K. M. An Alkyne Hydrosilylation-Oxidation Strategy for the Selective Installation of Oxygen Functionality. J. Am. Chem. Soc. 2005, 127, 10028-10038. (j) Shan, M.; Kishi, Y. Concise and Highly Stereoselective Synthesis of the C20-C26 Building Block of Halichondrins and Eribulin. Org. Lett. 2012, 14, 660-663. (k) Volchkov, I.; Lee, D. Asymmetric Total Synthesis of (-)-Amphidinolide V Through Effective Combinations of Catalytic Transformations. J. Am. Chem. Soc. 2013, 135, 5324-5327.

(11) (a) Trost, B. M.; Machacek, M. R.; Ball, Z. T. RutheniumCatalyzed Vinylsilane Synthesis and Cross-Coupling as a Selective Approach to Alkenes: Benzyldimethylsilyl as a Robust Vinylmetal Functionality. Org. Lett. 2003, 5, 1895-1898. (b) Ricci, A.; Degl'innocenti, A.; Fiorenza, M.; Taddei, M.; Spartera, M. A.; Walton, D. R. M. Fluoride Ion Induced Reactions of Organosilanes with Electrophiles. Tetrahedron Lett. 1982, 23, 577-578. (c) Gudmundsson, H. G.; Kuper, C. J.; Cornut, D.; Urbitsch, F.; Elbert, B. L.; Anderson, E. A. Synthesis of Cyclic Alkenyl Dimethylsiloxanes from Alkynyl Benzyldimethylsilanes and Application in Polyene Synthesis. J. Org. Chem. 2019, 84, 14868-14882. (d) Eaborn, C.; Parker, S. H. Organosilicon Compounds. Part XI. The Alkali-Catalysed Cleavage of Substitute Benzyltrimethylsilanes. J. Chem. Soc. 1955, 126-131.

(12) Oh, S. F.; Pillai, P. S.; Recchiuti, A.; Yang, R.; Serhan, C. N. Pro-resolving actions and stereoselective biosynthesis of $18 \mathrm{~S}$ E-series resolvins in human leukocytes and murine inflammation. J. Clin. Invest. 2011, 121, 569-581.

(13) (a) Verkruijsse, H. D.; Heus-Kloos, Y. A.; Brandsma, L. Efficient methods for the preparation of acetylenic ketones. J. Organomet. Chem. 1988, 338, 289-294. (b) Saito, S.; Tanaka, T.; Koizumi, T.; Tsuboya, N.; Itagaki, H.; Kawasaki, T.; Endo, S.; Yamamoto, Y. Nickel(0)-Catalyzed Unprecendented Zipper Annulation of Certain Conjugated Enynes. J. Am. Chem. Soc. 2000, 122, $1810-1811$. 
(14) Matsumura, K.; Hashiguchi, S.; Ikariya, T.; Noyori, R. Asymmetric Transfer Hydrogenation of Alpha, Beta-Acetylenic Ketones. J. Am. Chem. Soc. 1997, 119, 8738-8739.

(15) Acetylation of propargylic alcohols is beneficial for the selectivity of the subsequent semihydrogenation. See ref 11 a and: Panek, J. S.; Clark, T. D. Ireland-Claisen rearrangements of chiral (Z)vinylsilanes. Highly diastereoselective synthesis of anti- $\alpha$-alkoxy- $\beta$ (dimethylphenylsilyl)-(E)-hex-4-enoates. J. Org. Chem. 1992, 57, 4323-4326.

(16) Hirao, T.; Masunaga, T.; Ohshiro, Y.; Agawa, T. Reduction of gem-dibromides with diethyl phosphite. J. Org. Chem. 1981, 46, $3745-3747$.

(17) Abbas, S.; Hayes, C. J.; Worden, S. The "Hirao reduction" revisited: a procedure for the synthesis of terminal vinyl bromides by the reduction of 1,1-dibromoalkenes. Tetrahedron Lett. 2000, 41, 3215-3219.

(18) Hayashi, T.; Konishi, M.; Okamoto, Y.; Kabeta, K.; Kumada, M. Asymmetric synthesis catalyzed by chiral ferrocenylphosphinetransition metal complexes. 3. Preparation of optically active allylsilanes by palladium-catalyzed asymmetric Grignard crosscoupling. J. Org. Chem. 1986, 51, 3772-3781.

(19) Soullez, D.; Plé, G.; Duhamel, L. $\omega$-Halogeno Polyenals: Preparation and Application to a One-pot Synthesis of Polyenals from Carbonyl Compounds. J. Chem. Soc., Perkin Trans. 1 1997, 16391646.

(20) (a) Gao, Y.; Klunder, J. M.; Hanson, R. M.; Masamune, H.; Ko, S. Y.; Sharpless, K. B. Catalytic asymmetric epoxidation and kinetic resolution: modified procedures including in situ derivatization. J. Am. Chem. Soc. 1987, 109, 5765-5780. (b) Martin, V. S.; Woodard, S. S.; Katsuki, T.; Yamada, Y.; Ikeda, M.; Sharpless, K. B. Kinetic resolution of racemic allylic alcohols by enantioselective epoxidation. A route to substances of absolute enantiomeric purity? J. Am. Chem. Soc. 1981, 103, 6237-6240.

(21) (a) Rodríguez, A.; Nomen, M.; Spur, B. W.; Godfroid, J.-J. Effective Stereoselective Total Synthesis of 16,16-Dimethyl Prostaglandin E2. Arch. Pharm. 1998, 331, 279-282. (b) Rodriguez, A. R.; Spur, B. W. First total synthesis of the anti-inflammatory lipid mediator Resolvin D6. Tetrahedron Lett. 2012, 53, 86-89.

(22) Ichige, T.; Matsuda, D.; Nakata, M. Stereoselective synthesis of (E)- and (Z)-3-hydroxy-2-methyl-1-alkenyl iodides by base-promoted ring-opening of iodomethylated epoxides. Tetrahedron Lett. 2006, 47, $4843-4848$.

(23) A one-step synthesis of $\mathbf{2 7}$ has been described; see: Cahiez, C.; Alexakis, A.; Normant, J. F. A Highly Stereoselective Preparation of 1,4- and 1,5-Alkadienes. Synthesis 1978, 1978, 528-530 (because of restrictions in the use of acetylene, an alternative route was followed, as described in the Supporting Information).

(24) See the Supporting Information for details of structural assignment.

(25) This $\gamma$-lactone was isolated as a mixture of alkene stereoisomers, which may be due to a reversible intramolecular Tsuji-Trost reaction. This prevented productive hydrolysis/recycling of this compound. The formation of a $\gamma$-lactone accompanied the reaction under all conditions screened.

(26) Spectroscopic data for synthetic resolvin D3 was identical to that reported in ref 6 a.

(27) Spectroscopic data for synthetic resolvin E1 was identical to that reported in ref $8 \mathrm{~d}$. 